\title{
Brain mast cells could be a potential therapy for the neruoaids diseases
}

\section{Opinion}

In the brain especially the central nervous system may infected by a variety of microbes Dropp. ${ }^{1}$ Here the main concern is the immune response in the CNS is limited and access to the antimicrobial agent is also limited. So the neuroinfection of AIDS should be combated with the aids of the available immune system and complement system inside the brain, because the neural infection are life threatening. There is an urgent need of therapy for neuroinfectious disease. The molecular and cellular level work has shown the link between host response to pathogens, microglia crosstalk, and pathobiology of neuroinflammation. Brain mast cells are multipotent cells which plays a an immense sentinel role in host defense against microorganisms such as parasites, bacteria, fungi and viruses and take part in the innate and adaptive immune responses of the human body. Research finding revealed a major link between the neurons and inflammation via neuropeptides Tore \& Tuncel, ${ }^{2}$ which induces the brain mast cells degranulation and chemokine production. These neuropeptides shows direct and indirect effects on brain mast cells like upregulating or downregulating the degranulation, triggering, modulation, or amplification of mediator content and release. By exploring the interactions of mast cells and neurons will pay a new path for therapy for neuroinfectious AIDS. This interaction is mainly caused by the neuropeptides; this interaction can make a better understanding of the autocrine, paracrine, and neuro-immune-endocrine systems in physiology and physiopathology.

My opinion is in the future research may be concentrated much on the mast cell manipulator drug designs, which can modulate the mast cell granular content, which has an selective effect on mediators

\author{
Volume 3 Issue 5 - 2016
}

\author{
Satheesh Natarajan \\ School of Health Sciences, University of Kwazulu Natal, South \\ Africa
}

Correspondence: Satheesh Natarajan, CPRU, School of Health Sciences, University of Kwazulu Natal,Westville campus, Durban, South Africa, Tel +2740660259,

Email satheesh.sahana@gmail.com

Received: May 24, 2016 | Published: June 16, 2016

release, activity and, ablation of mast cells. In short understanding the neuropeptides and its interaction with the brain mast cells can lead to the development of a novel therapeutic approach to treat neuro AIDS.

\section{Acknowledgements}

None.

\section{Conflict of interest}

The author declares no conflict of interest.

\section{References}

1. Dropp JJ. Mast cells in mammalian brain. Acta Anat (Basel). 1976;94(1):1-21.

2. Tore F, Tuncel N. Mast cells: target and source of neuropeptides. Curr Pharm Des. 2009;15(29):3433-3445. 